\title{
MOVING AVERAGE RULES AS A SOURCE OF MARKET INSTABILITY
}

\author{
CARL CHIARELLA*, XUE-ZHONG HE* AND CARS HOMMES** \\ *School of Finance and Economics \\ University of Technology, Sydney \\ PO Box 123 Broadway \\ NSW 2007, Australia \\ **CeNDEF \\ Faculty of Economics and Econometrics \\ University of Amsterdam \\ Roetersstraat 11, NL-1018 WB \\ Amsterdam, The Netherlands
}

\begin{abstract}
Despite the pervasiveness of the efficient markets paradigm in the academic finance literature, the use of various moving average (MA) trading rules remains popular with financial market practitioners. This paper proposes a stochastic dynamic financial market model in which demand for traded assets has both a fundamentalist and a chartist component. The chartist demand is governed by the difference between current price and a (long-run) MA. Our simulations show that the MA is a source of market instability, and the interaction of the MA and market noises can lead to the tendency for the market price to take long excursions away from the fundamental. The model reveals various market price phenomena, the coexistence of apparent market efficiency and a large chartist component, price resistance levels, long memory and skewness and kurtosis of returns.
\end{abstract}

JEL classifications: D83, D84, E21, E32, C60.

Keywords: Moving Averages, Fundamentalists, Trend Followers, Stability, Evolutionary Switching.

Despite all the evidence presented in academic journals that security prices follow random walks, and consequently that these security markets are at least weak-form efficient, as defined in [10], the use of technical trading rules still seems to be widespread amongst financial market practitioners. Technical analysts, also known as "chartists", attempt to forecast future prices by the study of patterns of past prices and a few other related summary statistics about security trading. Basically, they believe that shifts in supply and demand can be detected in charts of market movements. There have been various studies (see $[1,2,4,15]$ ) of the use and profitability of technical analysis, we refer the reader to [14] for an up-to-date survey.

If one remains within the efficient market paradigm there is little scope to introduce apparently "irrational" traders using technical analysis. One strand of literature that does leave some room for such "irrational" agents is that of heterogeneous agent models (HAMs) of financial markets, see for example [9, 5, 3, 6, 7] and the recent surveys [11, 12] and the many references therein. [8] have recently proposed a simple behavioural HAM with a group of fundamentalists

This work was initiated while Tony He was visiting CeNDEF, whose hospitality he gratefully acknowledges. This paper has benefitted from comments of seminar participants at the CEF-conference, Amsterdam, July 2004 and the Econophysics conference, Canberra, November 2005. The usual caveat applies. Financial support from the Netherlands Organization of Scientific Research (NWO) under a NWO-MaG Pionier grant, the Australian Research Council (ARC) under a Discovery Grant (DP0450526), and the University of Technology, Sydney under a Research Excellence Grant are also gratefully acknowledged. Corresponding author: Xuezhong (Tony) He, Email: Tony.He1@uts.edu.au. Ph: (61 2) 9514 7726. Fax: (61 2) 95147711. 
and a group of chartists using a (long-run) moving average (MA) rule similar to the rules used in financial practice. The technical analysts are assumed to react to buy-sell signals generated by the difference between a long-run and a short-run MA. Both types of traders are boundedly rational in the sense that, based on a fitness measure given by realized capital gains, traders switch from strategies with low fitness to ones with high fitness. It is found that the stability properties of the underlying deterministic model can be characterized by the reaction coefficient of the fundamentalists, the extrapolation rate of the chartists, the intensity of choice to switching strategies, and the lag length used for the MA. This paper extends the analysis to the corresponding stochastic model and analyze the stability properties and dynamic behaviour of the model, particularly in relation to the MA trading strategies, and the potential for the model to generate phenomenon such as long deviations of the price from its fundamental, the coexistence of apparent market efficiency and a large chartist component, price resistance levels, long memory and skewness and kurtosis of returns.

The plan of the paper is as follows. We first introduce the model and briefly review the local stability and bifurcations of the fundamental steady state of the deterministic model. We then introduce stochastic fundamental price and noise-trader demand processes, and examine the effect of these noise processes when the prices of the corresponding deterministic system are switching between bull and bear markets. This non-linear stochastic model illustrates a range of phenomena observed in real markets.

Consider an asset pricing model with only one risky asset. Let $P_{t}$ be the price (cum dividend) per share of the risky asset at time $t$. Let $n_{h, t}$ be the market fraction of type $h$ traders at time $t$ with $h=f$ (fundamentalists) and $c$ (chartists) and $n_{f, t}+n_{c, t}=1$. Let the excess demand for the risky asset of representative trader of type $h$ at time $t$ be $D_{t}^{h}$. Then the population weighted aggregate excess demand at time $t$ is given by $D_{t}=n_{f, t} D_{t}^{f}+n_{c, t} D_{t}^{c}$. We assume that prices are set each period via a market maker who adjusts the price according to

$$
P_{t+1}=P_{t}+\tilde{D}_{t}+\mu D_{t}=P_{t}+\tilde{D}_{t}+\mu\left[n_{f, t} D_{t}^{f}+n_{c, t} D_{t}^{c}\right],
$$

where $\tilde{D}_{t} \sim \mathcal{N}\left(0, \sigma_{t}^{2}\right)$ captures a random excess demand process either driven by unexpected news about fundamentals, or representing noise created by noise traders, $\sigma_{t} \geq 0$ and the parameter $\mu>0$ measures the speed of price adjustment (or the aggregate risk tolerance) of the market maker to the excess demand.

The fundamentalists believe that the market price should be given by the fundamental price that they have estimated based on various types of fundamental information, such as earnings, general economic forecasts and so forth. They buy/sell the stock when the current price is below/above the fundamental price. For simplicity, we first assume that ${ }^{1}$ the fundamental price is a positive constant $P^{*}$ and the average excess demand of the fundamentalists is given by $D_{t}^{f}=\alpha\left(P^{*}-P_{t}\right)$, where the parameter $\alpha>0$ is a combined measure of the aggregate risk tolerance of the fundamentalists and their reaction to the mis-pricing.

For the technical analysts, their average excess demands are assumed to be governed by $D_{t}^{c}=\tanh \left(a \psi_{t}^{L}\right)$, where $a>0$ is a constant, and $\psi_{t}^{L}=P_{t}-m a_{t}^{L}$ defines a trading signal, the difference between the current price and a moving average (MA) $m a_{t}^{L}=(1 / L) \sum_{i=0}^{L-1} P_{t-i}$. This captures one of the very popular technical trading rules whereby technical analysts wish to be long (short) when the current price is above (below) the MA. When $a$ is small, the technical analysts initially react cautiously to the long/short signals, in a sense waiting to confirm the maintenance of the change in sign of the signal. In this way they minimize the costs incurred if the signal changes frequently in a short time period. It also captures the limited long/short positions, risk averting behaviour and traders' budget constraints.

\footnotetext{
${ }^{1}$ A constant fundamental price is assumed for our analysis of the deterministic model, while a random walk fundamental price will be introduced for the stochastic version of the model.
} 
Following the mechanism in [3], we define the fitness functions $\pi_{f, t}, \pi_{c, t}$ as the realized net profits of each group, $\pi_{f, t}=D_{t-1}^{f}\left(P_{t}-P_{t-1}\right)-C_{f}, \pi_{c, t}=D_{t-1}^{c}\left(P_{t}-P_{t-1}\right)-C_{c}$, where $C_{f}, C_{c} \geq 0$ are the costs of the respective strategies. The population fractions are then updated by the well known logit model probabilities (see, e.g. [13])

$$
n_{f, t}=\frac{e^{\beta U_{f, t}}}{e^{\beta U_{f, t}}+e^{\beta U_{c, t}}}, \quad n_{c, t}=\frac{e^{\beta U_{c, t}}}{e^{\beta U_{f, t}}+e^{\beta U_{c, t}}},
$$

where $U_{f, t}=\pi_{f, t}+\eta U_{f, t-1}, \quad U_{c, t}=\pi_{c, t}+\eta U_{c, t-1}$. Here $\beta \geq 0$ is the intensity of choice measuring how quickly agents switch between the two strategies. In particular, if $\beta=0$, there is no switching between strategies, while for $\beta=\infty$ all agents immediately switch to the best strategy. Through the parameter $\eta$ the $U_{f, t}, U_{c, t}$ are effectively geometrically declining weighted averages of past realized profits.

Set $m_{t}=n_{f, t}-n_{c, t}$. Based on the above analysis, the market price of the risky asset is determined according to

$$
P_{t+1}=P_{t}+\tilde{D}_{t}+\frac{\mu}{2}\left[\left(1+m_{t}\right) \alpha\left(P^{*}-P_{t}\right)+\left(1-m_{t}\right) \tanh \left[a\left(P_{t}-m a_{t}^{L}\right)\right]\right]
$$

and, from (2), the difference of population fractions $m_{t}$ evolves according to

$$
m_{t}=\tanh \left[\frac{\beta}{2}\left(U_{t}-C\right)\right], \quad U_{t}=\left[D_{t-1}^{f}-D_{t-1}^{c}\right]\left[P_{t}-P_{t-1}\right]+\eta U_{t-1},
$$

where $C=C_{f}-C_{c}$ will be positive if we assume that the fundamentalists incur greater costs than the chartists. By setting $\sigma_{t}=0$, (3) becomes a nonlinear deterministic equation of the price

$$
P_{t+1}=P_{t}+\frac{\mu}{2}\left[\left(1+m_{t}\right) \alpha\left(P^{*}-P_{t}\right)+\left(1-m_{t}\right) \tanh \left[a\left(P_{t}-m a_{t}^{L}\right)\right]\right] .
$$

The dynamics of the deterministic model (4)-(5) is analyzed in [8]. To guide our analysis on the price dynamics of the stochastic model, we briefly review the main results in [8]. For the deterministic model, the fundamental price $P^{*}$ is the unique steady state price and different lag lengths $L$ of the MA rules play different roles on the stability of the fundamental price. Intuitively we would expect the fundamentalists to represent a stabilizing force and the activities of the chartists to destabilize an otherwise stable market price, and an increase of the lag length might enlarge the stability region. The stability analysis in [8] shows that increasing the lag length $L$ in the end destabilizes the system in general. This is a very interesting result and the intuition for this instability result is the following. Chartist demand depends on the difference between the long-run MA and the current price. As $L$ increases, the MA becomes smoother and more sluggish. When the relative effect of chartists at the steady state is bigger than that of fundamentalists, a small change in the price leads to a relatively large increase of chartists demand to destabilizing the price.

As an illustrative example, Fig. 1 illustrates how the phase plot (in terms of $\left(P_{t}, m_{t}\right)$ ) changes as the lag length $L$ increases, where the fundamental price is locally stable for $L=2,3,4$, but it is unstable for $L \geq 5$. One can see that, as $L$ increases, the size of the attractor is enlarged, implying that the deviations of both price and population from the fundamental value are enlarged. Hence an increase in the MA window $L$ destabilizes the price dynamics. This destabilizing effect becomes more significant when $L$ is increased further to $L=9,10,50$ and the price dynamics become even more complicated for $L=90$ and 100 , as indicated by the phase plots in Fig. 1. This demonstrates that for large lag lengths the price can take large excursion from the fundamental and at turning points in the price, the fraction of fundamentalists/ chartists can change dramatically.

We now use numerical simulations to attempt to gain some insights into the different behaviour of the nonlinear stochastic model with stochasticity arising from noise created by the 

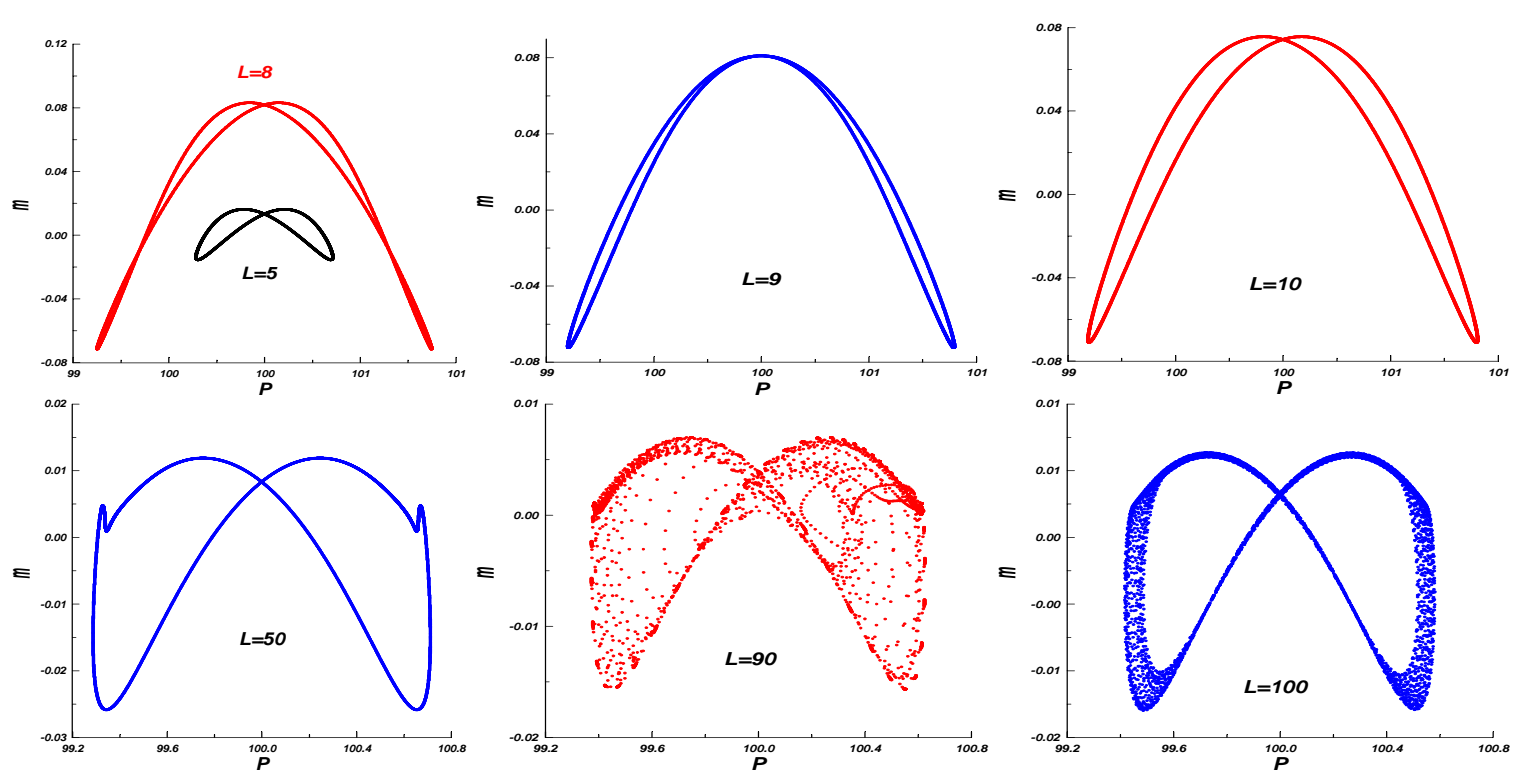

Figure 1. Phase plots of $\left(m_{t}, P_{t}\right)$ for fixed $\beta=0.4$ and various $L=$ $5,8,9,10,50,90$ and 100, where $\alpha=1, \mu=2, \beta=0.4, \eta=0.2, a=1, C=0$.

noise traders and a noisy fundamental. We assume that the fundamental price follows the random walk $P_{t+1}^{*}=P_{t}^{*}\left[1+\sigma_{\delta} \delta_{t}\right]$, where $\sigma_{\delta} \geq 0$ is a constant measuring the volatility of the return and $\delta_{t} \sim \mathcal{N}(0,1)$. Notice that this specification ensures that relative fundamental price changes are stationary. In addition to a noisy fundamental, we consider two different forms for $\tilde{D}_{t}$ representing noisy excess demand created by noise traders in (1).
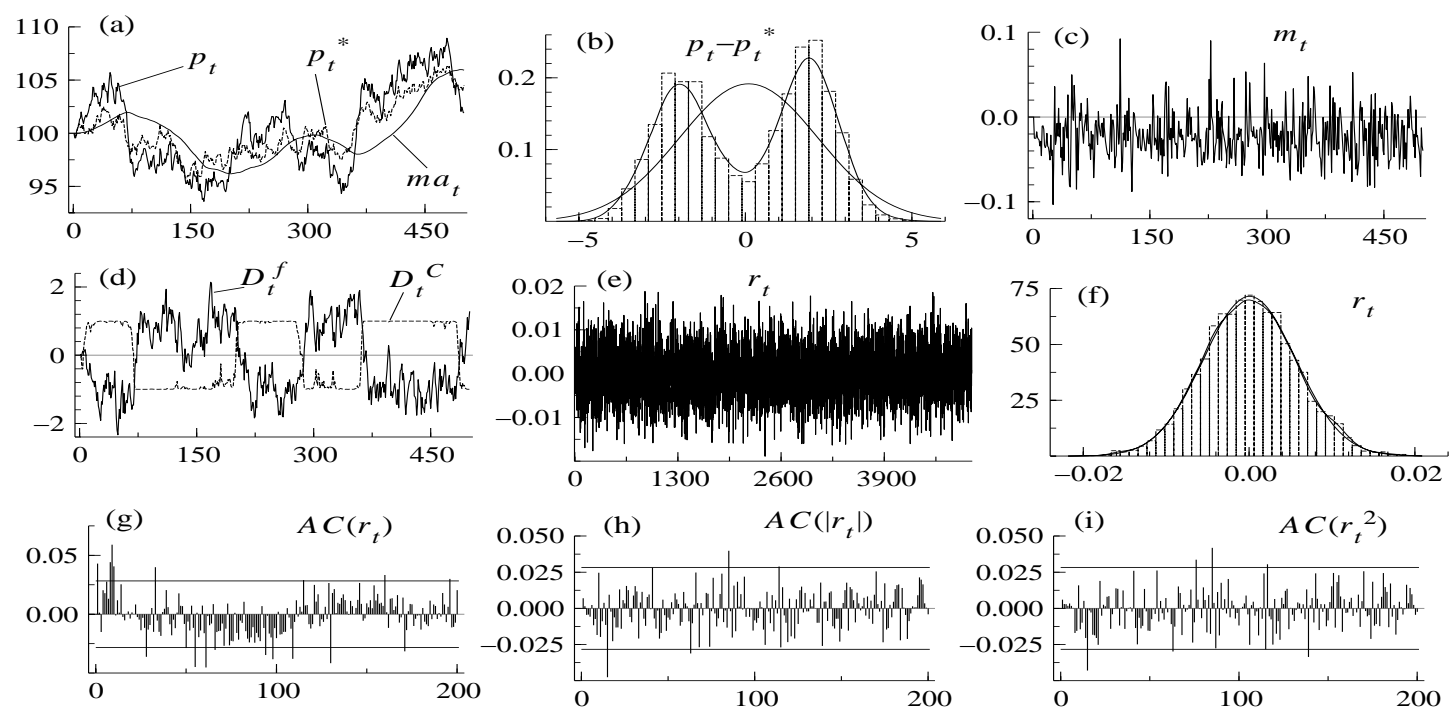

FIGURE 2. Time series, density distributions, and the autocorrelation coefficients (ACs) of the returns, absolute and squared returns for $L=100$ and $\alpha=0.5, \beta=0.05, \mu=1, \eta=0.2, a=1, C=1, \sigma_{\delta}=5 \%$. Here the noise demand is proportional to the market price $\tilde{D}_{t}=\sigma_{\epsilon} \epsilon_{t} P_{t}$ with $\sigma_{\epsilon}=0.5 \%$.

We consider first $\tilde{D}_{t}=\sigma_{\epsilon} \epsilon_{t} P_{t}$, that is the size of the noise depends on the current market price. For the given set of parameters, Fig. 2(a) and (d) show that the market price follows the fundamental price in general, although it is above (below) the fundamental price when the technical analysts take long (short) positions and the market is dominated by technical analysts. Most of the time, the fundamentalists and trend followers take opposite positions. However, 
following the cross-overs of the market price and the moving average, there are very short transition periods where they both take the same position. This immediately pushes the market price away from the fundamental price over long time periods (close to 100 days). This is clearly indicated by the bi-model distribution of the difference $p_{t}-p_{t}^{*}$ in Fig. 2 (b). In fact, this bi-model distribution feature is robust for various combinations of parameters and noise sizes and is dominated by the bi-model distribution of the underlying price dynamics of the deterministic model. In addition, it is found from Fig. 2 (e)-(i) that the returns $r_{t}$ are close to being normally distributed with no significant autocorrelation coefficients (ACs) for the raw returns, the absolute and squared returns. This means that the market can appear to be quite efficient even though it is dominated by technical analysts and the market price is consistently pushed away from the fundamental price. This is a very interesting observation. We would suggest that Fig. 2 provides a basis for the existence of upper and lower price resistance levels in an apparent efficient market. These resistance levels are often referred to in the practitioner literature but are scorned by advocates of the efficient markets paradigm.
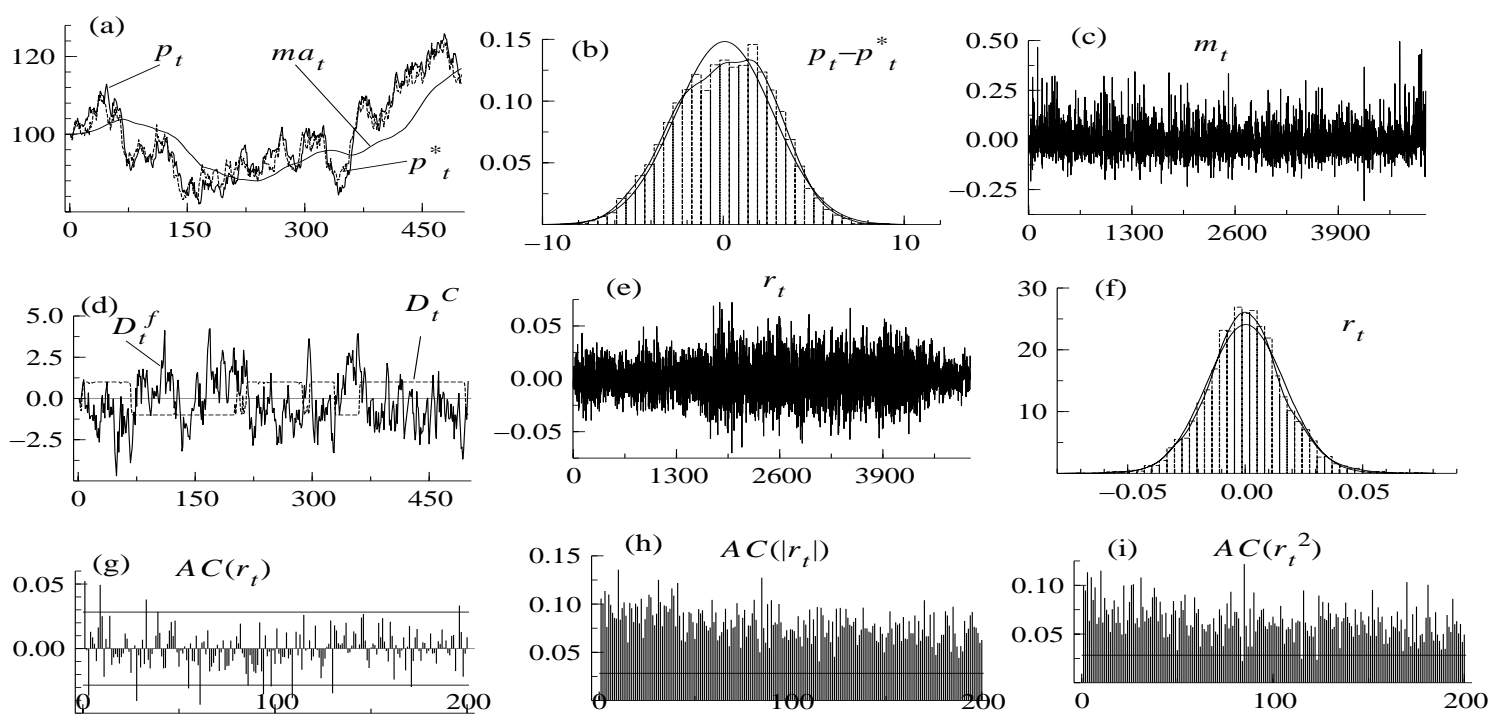

FIgURE 3. Time series, density distributions, and the autocorrelation coefficients (ACs) of the returns, absolute and squared returns for $L=100$ and $\alpha=0.5, \beta=0.05, \mu=1, \eta=0.2, a=1, C=1, \sigma_{\delta}=20 \%$. Here the noise demand is independent from the market price $\tilde{D}_{t}=\sigma_{\epsilon} \epsilon_{t}$ with $\sigma_{\epsilon}=1$.

An important stylized fact of financial markets is that of long memory. This is characterized by insignificant ACs for the raw returns but significant ACs for the absolute and squared returns. It would be interesting to know if the model is able to generate such long memory behaviour. We now consider the second form of the noisy demand $\tilde{D}_{t}=\sigma_{\epsilon} \epsilon_{t}$, which is independent from the market price. With this choice, Fig. 3 illustrates the corresponding time series, distributions, and ACs for $\sigma_{\delta}=20 \%$ for a fixed $\sigma_{\epsilon}=1$ (which is about $1 \%$ of the fundamental value). In this case, the bi-model distribution observed in Fig. 2 (b) disappears. For small $\sigma_{\delta}$, for example $5 \%$, it is found that the fundamental price is less volatile and the market returns are close to a normal distribution. However, for $\sigma_{\delta}=20 \%$, the fundamental price is more volatile, and Fig. 3 shows a non-normal distribution of the market returns with some skewness and high kurtosis. Furthermore, the AC patterns of the market returns in Fig. 3 (B) (g)-(i) show insignificant ACs for the returns but significant ACs for the absolute and squared returns. This indicates, when the noisy demand is independent from the market price, a high volatility of the fundamental price can reduce the market efficiency, generate the long memory feature as well as the skewness and kurtosis observed in asset returns. 
Based on the analysis above, we observe some interesting phenomena. (i) When the fundamental price follows a stochastic process, the market price closely follows the fundamental price. (ii) The switching between bull and bear markets happens when both types of traders take the same position, a very intuitive result. (iii) Different forms of the noise demand created by the noise traders can have a different impact on the price dynamics. When the noise trader demand is proportional to the market price, the price dynamics are dominated by the underlying price dynamics of the deterministic model. In addition, the market can appear efficient even though the market is dominated by technical analysts and the market price is consistently pushed away from the fundamental price. When the noise trader demand is independent of the market price, some stylized facts, including skewness, high kurtosis, and possible long memory, can be generated when the volatility of the fundamental price is high. In future research on models of the type presented here there is a need to analyse in more depth the interaction of the non-linear and stochastic elements.

\section{REFERENCES}

[1] Allen, H. and M. Taylor 'Charts, noise and fundamentals in the London foreign exvhange market', Economic Journal 100, 49-59, 1990. Conference.

[2] Blume, L., Easley, D. and M. O'Hara , 'Market statistics and technical analysis: the role of volume', Journal of Finance 49, 155-181, 1994.

[3] Brock, W. and C. Hommes, 'Heterogeneous beliefs and routes to chaos in a simple asset pricing model', Journal of Economic Dynamics and Control 22, 1235-1274, 1998.

[4] Brock, W., Lakonishok, J. and B. LeBaron, 'Simple technical trading volatility and the stochastic properties of stock returns', Journal of Finance 47, 1731-1764, 1992.

[5] Chiarella, C. , 'The dynamics of speculative behaviour', Annals of Operations Research 37, 101-123, 1992.

[6] Chiarella, C. and X. He, 'Heterogeneous beliefs, risk and learning in a simple asset pricing model', Computational Economics 19, 95-132, 2002.

[7] Chiarella, C. and X. He, 'Heterogeneous beliefs, risk and learning in a simple asset pricing model with a market maker', Macroeconomic Dynamics 7, 503-536, 2003.

[8] Chiarella, C., He, X. and C. Hommes, 'A dynamic analysis of moving average rules', Journal of Economic Dynamics and Control, 2006, in press.

[9] Day, R. and W. Huang, 'Bulls, bears and market sheep', Journal of Economic Behavior and Organization 14, 299-329, 1990.

[10] Fama, E., 'Efficient capital markets: a review of theory and empirical work', Journal of Finance 25, 383-423, 1970.

[11] Hommes, C., "Heterogeneous Agent Models in Economics and Finance", chapter in: Tesfatsion, L. and Judd, K.L. (eds.) Handbook of Computational Economics Vol. 2: Agent-based Computational Economics, North-Holland, 2006, to appear.

[12] LeBaron, B., "Agent-based Computational Finance”, chapter in: Tesfatsion, L. and Judd, K.L. (eds.) Handbook of Computational Economics Vol. 2: Agent-based Computational Economics, North-Holland, 2006, to appear.

[13] Manski, C. and D. McFadden, Structural Analysis of Discrete Data with Econometric Applications, MIT Press, 1981.

[14] Park, C.-H. and Scott H. Irwin, 'The profitability of technical analysis: A review', AgMAS Project Research Report 2004-04

[15] Pring, M., Technical Analysis Explained, 3rd edn, McGraw-Hill, New York, 1991. 